\title{
Expression and significance of glucose transporter-1, P-glycoprotein, multidrug resistance-associated protein and glutathione $S$-transferase- $\pi$ in laryngeal carcinoma
}

\author{
ZHONG-PING MAO ${ }^{1}$, LI-JUN ZHAO ${ }^{1}$, SHUI-HONG ZHOU ${ }^{2}$, \\ MENG-QIN LIU ${ }^{3}$, WEI-FENG TAN ${ }^{3}$ and HONG-TIAN YAO ${ }^{4}$ \\ ${ }^{1}$ Department of Otolaryngology, The Second Hospital of Shaoxing City, Shaoxing, Zhejiang 312000; \\ ${ }^{2}$ Department of Otolaryngology, The First Affiliated Hospital, College of Medicine, Zhejiang University, Hangzhou, \\ Zhejiang 310003; ${ }^{3}$ Department of Pathology, The Second Hospital of Shaoxing City, Shaoxing, Zhejiang 312000; \\ ${ }^{4}$ Department of Pathology, The First Affiliated Hospital, College of Medicine, \\ Zhejiang University, Hangzhou, Zhejiang 310003, P.R. China
}

Received March 2, 2014; Accepted November 7, 2014

DOI: $10.3892 / 01.2014 .2752$

\begin{abstract}
Increasing glucose transporter-1 (GLUT-1) activity is one of the most important ways to increase the cellular influx of glucose. We previously demonstrated that increased GLUT-1 expression was an independent predictor of survival in patients with laryngeal carcinoma. Thus, GLUT-1 may present a novel therapeutic target in laryngeal carcinoma. In this study, the expression of GLUT-1, P-glycoprotein (P-gp), multidrug resistance-associated protein 1 (MRP1) and glutathione S-transferase- $\pi$ (GST- $\pi$ ) in laryngeal carcinomas was investigated by immunohistochemistry. Additionally, possible correlations between GLUT-1 and P-gp, MRP1 and GST- $\pi$ and various clinicopathological parameters were analyzed. In this study, 52.9\% (18/34), 58.8\% (20/34), $20.6 \%$ (7/34) and $58.8 \%$ (20/34) of the laryngeal carcinomas were positive for GLUT-1, P-gp, MRP1 and GST- $\pi$, respectively. The expression of GLUT-1, P-gp, MRP1 and GST- $\pi$ was higher in laryngeal carcinoma specimens when compared with laryngeal precancerous lesions $(\mathrm{P}<0.05)$. Pearson's correlation analysis showed correlations between GLUT-1 and P-gp $(\mathrm{r}=0.364$; $\mathrm{P}=0.034)$, GLUT-1 and MRP1 $(\mathrm{r}=0.359 ; \mathrm{P}=0.037)$ and $\mathrm{P}-\mathrm{gp}$ and GST- $\pi(r=0.426 ; P=0.012)$. GLUT-1 expression was found to significantly correlate with tumor-node-metastasis classification $(\mathrm{P}=0.02)$ and clinical stage $(\mathrm{P}=0.037)$. Furthermore, $\mathrm{P}-\mathrm{gp}$ was found to significantly correlate with clinical stage
\end{abstract}

Correspondence to: Professor Shui-Hong Zhou, Department of Otolaryngology, The First Affiliated Hospital, College of Medicine, Zhejiang University, 79 Qingchun Road, Hangzhou, Zhejiang 310003, P.R. China

E-mail: zhouyunzhoush@163.com

Key words: laryngeal carcinoma, glucose transporter-1, P-glycoprotein, multidrug resistance-associated protein, glutathione-s-transferase- $\pi$
$(\mathrm{P}=0.026)$. Univariate analysis showed that MRP1 expression was significantly associated with poor survival $\left(c^{2}=5.16\right.$; $\mathrm{P}=0.023)$. Multivariate analysis revealed that lymph node metastasis $(\mathrm{P}=0.009)$ and MRP1 overexpression $(\mathrm{P}=0.023)$ were significant predictors of poor survival. In the present study, the expression of GLUT-1, P-gp, MRP1 and GST- $\pi$ in laryngeal carcinomas was investigated, as well as the correlations between these proteins. P-gp was found to significantly correlate with clinical stage, while MRP1 overexpression was significantly associated with poor survival.

\section{Introduction}

The mechanism of carcinoma resistance to chemoradiotherapy may involve multiple factors. Among these, hypoxia is an important factor in the chemoresistance of head and neck carcinomas, such as oral squamous cell carcinoma and nasopharyngeal carcinoma $(1,2)$. Under conditions of hypoxic stress, carcinoma cells require more energy to support cell proliferation. Glucose is an important source of energy. Therefore, increasing glucose transporter-1 (GLUT-1) activity is one of the most important ways to increase the cellular influx of glucose (3). In our previous study, it was demonstrated that increased GLUT-1 expression was an independent predictor of survival in patients with laryngeal carcinoma (4). Thus, GLUT-1 may present a novel therapeutic target in laryngeal carcinoma $(5,6)$. However, few studies have investigated GLUT-1 expression and tumor drug resistance (7-10).

Another important factor in tumor resistance to chemotherapy is intrinsic chemotherapy resistance (11-14). Various drug transporter proteins inside tumor cells are involved in intrinsic chemotherapy resistance, including P-glycoprotein (P-gp), multidrug resistance-associated protein (MRP) and glutathione-s-transferase- $\pi$ (GST- $\pi$ ). These drug transporters are overexpressed in a number of cancer types, such as liver cancer, lung cancer, glioma and gallbladder cancer (11-14), and overexpression of these proteins is associated with hypoxia $(15,16)$. In the present study, the expression of GLUT-1, P-gp, MRP1 
and GST- $\pi$ in laryngeal carcinomas was investigated by immunohistochemistry (IHC). The present study investigated the correlations between the expression of these proteins, with respect to various clinical and pathological features of laryngeal carcinoma.

\section{Materials and methods}

Patients and tissues. A total of 34 paraffin-embedded archival tissue blocks from laryngeal squamous cell carcinoma patients were obtained from The Second Hospital of Shaoxing City (Shaoxing, China) between May 2005 and January 2012. A total of 34 paraffin-embedded archival tissue blocks from patients with precancerous lesions were also obtained from The First Affiliated Hospital, College of Medicine, Zhejiang University (Zhejiang, China). A representative paraffin block from each tumor was selected for immunohistochemical analysis. The diagnosis was confirmed after all hematoxylin and eosin-stained sections were reviewed blindly. No patients had received preoperative radiotherapy or chemotherapy. Demographic and clinicopathological data, including gender, age, tumor-node-metastasis (TNM) stage were retrospectively collected. The study protocol was approved by the institutional review board of The Second Hospital of Shaoxing City and The First Affiliated Hospital, College of Medicine, Zhejiang University and all patients provided consent.

IHC. Formalin-fixed and paraffin-embedded tissue blocks from primary lesions were cut into $4-\mu \mathrm{m}$ sections, and representative sections were analyzed immunohistochemically using an EliVision ${ }^{\mathrm{TM}}$ Plus IHC kit (Fuzhou Maixin Biotechnology Development Co., Ltd., Fuzhou, China) for GLUT-1 (cat. no. ab14683; 1:50) rabbit polyclonal; a mouse monoclonal antibody against P-gp (cat. no. ab3366; 1:100), a mouse monoclonal antibody against MRP1 (cat. no. ab63987; 1:100), and a mouse monoclonal antibody against GST- $\pi$ (cat. no. ab131059; 1:50; all antibodies purchased from Abcam, Cambridge, MA, USA). Primary antibodies were applied for $1 \mathrm{~h}$ at room temperature, and then sections were washed three times with $0.05 \mathrm{~mol} / \mathrm{l}$ Tris-buffered saline $(\mathrm{pH}$ 7.2) and incubated with $50 \mu 1$ of polymer enhancer (Fuzhou Maixin Biotechnology Development Co., Ltd.) for $20 \mathrm{~min}$. This was followed by incubation with $50 \mu$ l polymerized horseradish peroxidase-conjugated anti-mouse immunoglobulin G (Fuzhou Maixin Biotechnology Development Co., Ltd.) for $30 \mathrm{~min}$ at room temperature. GLUT-1 expression was considered positive if distinct membrane staining was identified. P-gp, MRP1, and GST- $\pi$ were identified in the membrane and/or cytoplasm. Protein analysis was performed in 10 random high-power fields; a total of 100 tumor cells were counted from each high-power field for each case and for all antibodies analyzed. The percentage of positive cells was calculated by dividing the number of positive tumor cells by the total number of tumor cells counted. Staining intensity was scored as follows: Negative staining (-), $<10 \%$ cells were stained positive; weak staining $(+), \geq 10$ but $<25 \%$ cells were stained positive; moderate staining $(++), \geq 25$ but $<75 \%$ cells were stained positive; and intense staining $(+++), 75-100 \%$ cells were stained positive.

Statistical analysis. Associations between GLUT-1, P-gp, MRP1 and GST- $\pi$ immunostaining and other parameters were analyzed using the $\chi^{2}$ and Fisher's exact tests. $\mathrm{P}<0.05$ was considered to indicate a statistically significant difference. The associations between GLUT-1 and P-gp, MRP1 and GST- $\pi$ were analyzed by Spearman's correlation. Overall survival, which was defined as the time from surgery until mortality from any cause, was plotted as a Kaplan-Meier curve. Univariate survival analysis was performed using the log-rank test and multivariate analysis was performed using Cox proportional-hazards regression analysis. All analyses were conducted using SPSS version 19.0 software (SPSS, Inc., Chicago, IL, USA).

\section{Results}

Patient characteristics. All patients had squamous cell carcinoma. The subjects included 33 males and one female with a mean age of 62.1 years (range, $45-76$ years). A total of 28 $(82.4 \%)$, five $(14.7 \%)$, and one $(2.9 \%)$ patients had tumors located in the glottis, supraglottis and subglottis, respectively. A total of 25 patients received partial laryngetomy (21 vertical partial laryngetomies and four supraglottic partial laryngetomies) and nine patients received total laryngetomy in addition to postoperative radiotherapy. TNM, clinical stage and other clinopathological parameters of the patients are shown in Table I. Six patients were lost to follow-up. Seven patients (20.6\%) developed local recurrence and two (5.9\%) developed distant metastases. Twenty-two patients were alive at the last follow-up (December 2012). The three- and five-year cumulative survival rates were 76.0 and $61.0 \%$, respectively.

Expression of GLUT-1, MRP1, P-gp and GST- $\pi$. In this study, 52.9 (18/34), 58.8 (20/34), 20.6 (7/34) and 58.8\% (20/34) of the laryngeal carcinomas were positive for GLUT-1, P-gp, MRP1 and GST- $\pi$, respectively (Fig. 1). Pearson's correlation analysis showed correlations between GLUT-1 and P-gp ( $r=0.364$; $\mathrm{P}=0.034)$, GLUT-1 and MRP1 ( $\mathrm{r}=0.359 ; \mathrm{P}=0.037)$, and $\mathrm{P}-\mathrm{gp}$ and GST- $\pi(\mathrm{r}=0.426 ; \mathrm{P}=0.012)$.

Association between GLUT-1, MRP1, P-gp and GST- $\pi$ expression in laryngeal carcinoma and clinicopathological parameters and prognosis. GLUT-1 expression was found to significantly correlate with TNM stage $(\mathrm{P}=0.02)$ and clinical stage $(\mathrm{P}=0.037)$. $\mathrm{P}$-gp was found to significantly correlate with clinical stage $(\mathrm{P}=0.026)$. No significant difference was identified between GLUT-1 and P-gp expression and the remaining clinicopathological factors investigated. No significant difference was identified between MRP1 and GST- $\pi$ expression and any of the clinicopathological factors investigated.

Univariate analysis showed that MRP1 expression was significantly associated with reduced survival $\left(\chi^{2}=5.16\right.$; $\mathrm{P}=0.023$; Fig. 2). By contrast, GLUT-1, P-gp and GST- $\pi$ expression were not associated with survival. Multivariate analysis revealed that lymph node metastasis $(\mathrm{P}=0.009)$ and MRP1 overexpression $(\mathrm{P}=0.023)$ were significant predictors of poor survival.

\section{Discussion}

P-gp, MRP1 and GST- $\pi$ are associated with intrinsic chemotherapy resistance (11-14). P-gp and MRP1, two important 


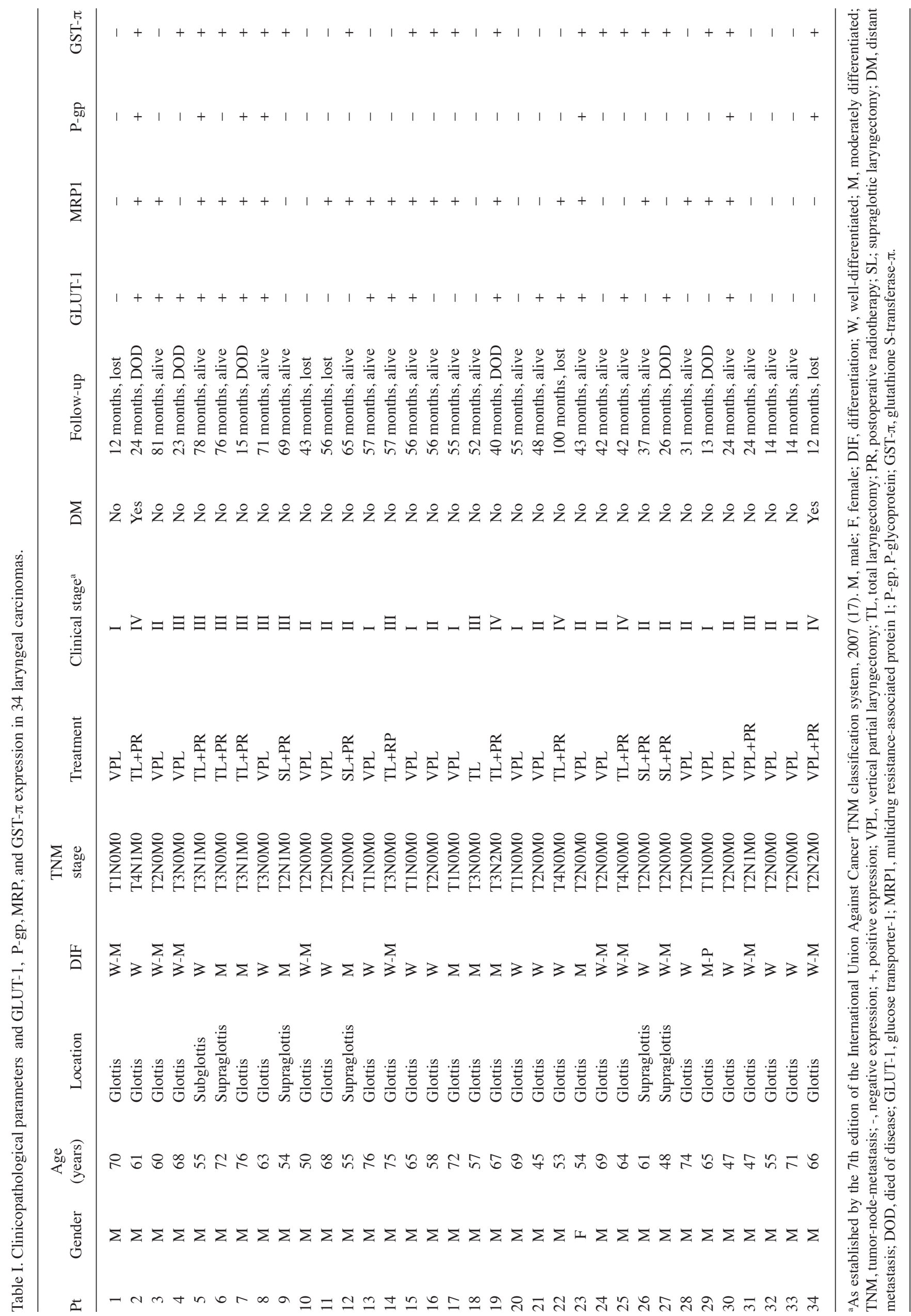



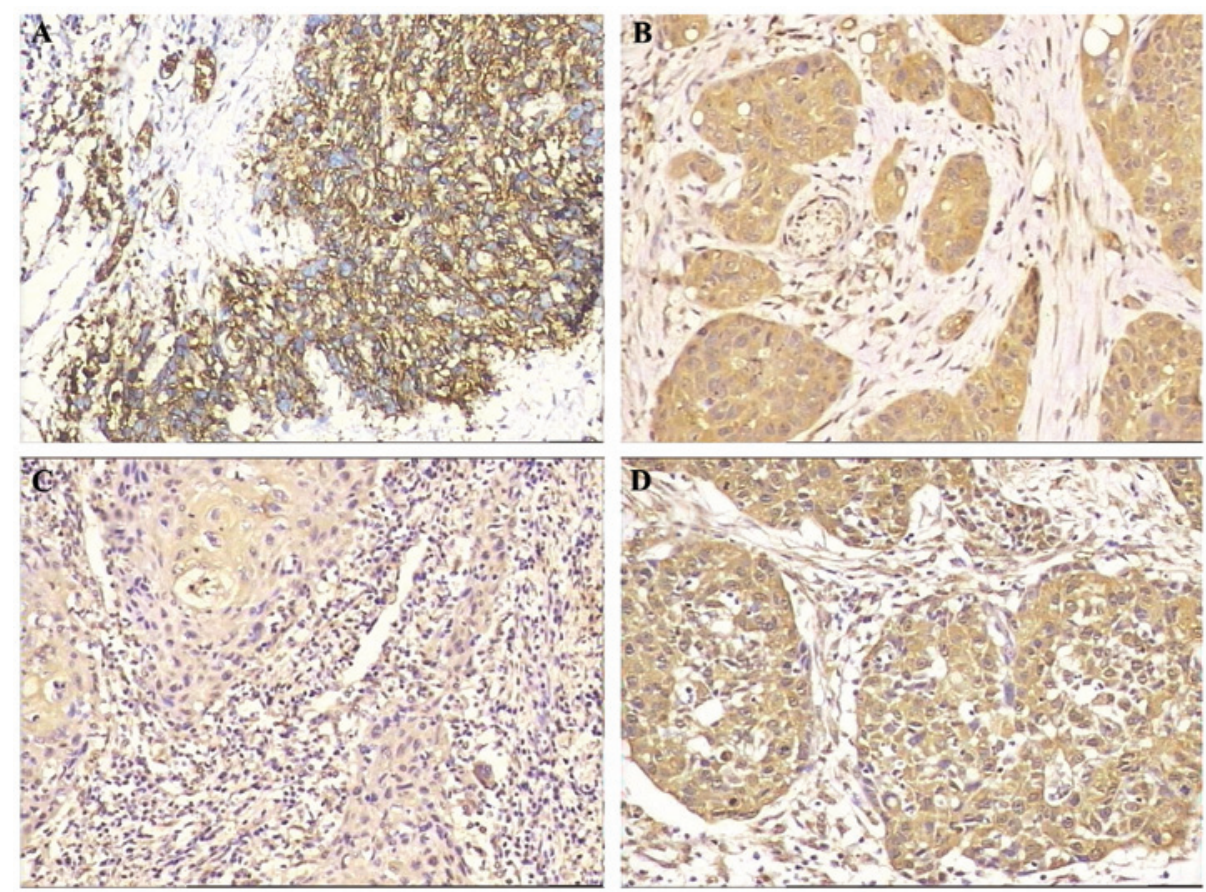

Figure 1. Immunohistochemical analysis of GLUT-1, P-gp, MRP1 and GST- $\pi$ protein expression in laryngeal carcinoma. Representative positive staining images for (A) Glut-1, (B) P-gp, (C) MRP1 and (D) GST- $\pi$. GLUT-1, glucose transporter-1; P-gp, P-glycoprotein; MRP1, multidrug resistance-associated protein 1 ; GST- $\pi$, glutathione S-transferase- $\pi$.

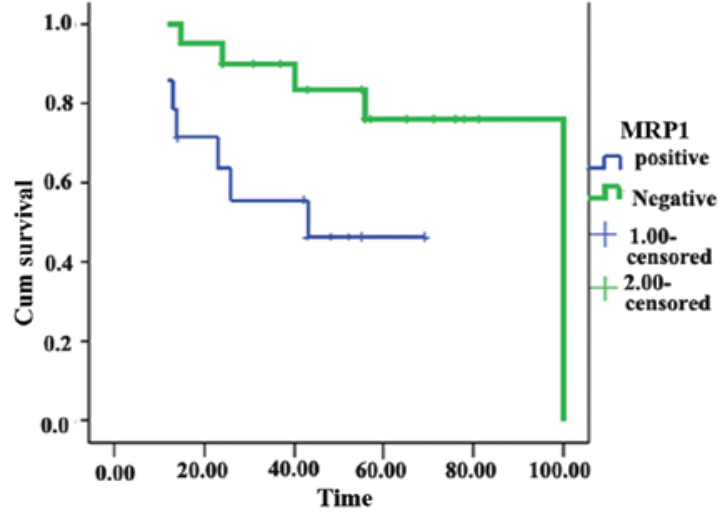

Figure 2. Univariate analysis shows that MRP1-positivity is significantly associated with poorer survival $\left(\chi^{2}=5.16 ; \mathrm{P}=0.023\right)$. MRP1, multidrug resistance-associated protein 1 .

ATP-binding cassette transporters, affect the intracellular drug concentration by altering drug influx or efflux (12). GST- $\pi$ is a member of the GST family, which catalyzes the conjugation of glutathione and leads to the inactivation of cytotoxic drugs $(12,13)$. The majority of studies have investigated P-gp, MRPs and GST- $\pi$ in human solid malignant tumors (11-14). In the present study, P-gp, MRP1 and GST- $\pi$ expression were investigated in laryngeal carcinomas. The expression of P-gp, MRP1 and GST- $\pi$ was higher than that in the laryngeal precancerous lesions $(\mathrm{P}<0.05)$. Among these proteins, $\mathrm{P}-\mathrm{gp}$ was found to significantly correlate with clinical stage $(\mathrm{P}=0.026)$ and MRP1 overexpression was significantly associated with poor survival $(\mathrm{P}=0.023)$. These results are similar to those for other human solid cancers. Yu et al (11) found that multidrug resistance protein 3 and MRP1 were poor prognostic factors in liver cancer. In four lung cancer cell lines, SK-MES-1, SPCA-1, NCI-H-460 and NCI-H-446, the expression of P-gp, MRP1 and GST- $\pi$ was different; the level of GST- $\pi$ in the SK-MES-1 cells was the highest, whereas the level of P-gp in the SPCA-1 cells was the lowest. The chemoresistance to cisplatin, doxorubicin and VP-16 in the four cell lines was also different; the SPCA-1 cell line was most resistant to cisplatin, and the SK-MES-1 cell line was most resistant to VP-16, but most sensitive to doxorubicin. There was a positive correlation between GST- $\pi$ expression and resistance to cisplatin, between TopoII $\alpha$ expression and resistance to VP-16, and a negative correlation was noted between TopoII $\alpha$ expression and resistance to doxorubicin. Among these proteins, GST- $\pi$ may be useful for the prediction of intrinsic resistance to cisplatin (12). P-gp and MDR have been found to be highly expressed in gallbladder carcinoma (14). Similarly, P-gp, MRP1 and GST- $\pi$ were highly expressed in gliomas (13). However, the regulatory mechanism underlying the high level of expression of these proteins in cancer remains unclear.

Previous studies have shown that the overexpression of these proteins may be associated with hypoxia $(15,16)$. Hypoxia is an important factor in chemoresistance $(1,2)$. A small number of studies have demonstrated co-expression of GLUT-1 and P-gp in the capillaries of the blood-brain barrier $(18,19)$. However, the association between GLUT-1, P-gp, MRP1 and GST- $\pi$ expression in human cancers has not been reported. In the present study, correlations were identified between GLUT-1 and P-gp, GLUT-1 and MRP1 and P-gp and GST- $\pi$ in laryngeal carcinoma.

In addition, GLUT-1 is associated with a poor response to chemoradiotherapy, and the silencing of GLUT-1 expression may increase sensitivity to chemotherapeutic agents (7-10). Solid cancers grow rapidly and cause hypoxia due to an insufficient 
supply of blood and oxygen. Under hypoxic conditions, GLUT-1 may supply glucose to meet the energy requirements of cancer cells (4,7-10). In the present study, high levels of GLUT-1 expression were identified in laryngeal carcinomas, which was similar to the results of our previous study regarding laryngeal carcinoma (4). However, GLUT-1 expression was not associated with any clinicopathological parameters. These results differ from our previous and other studies, and these differences may be due to variation in histopathological type, immunohistochemical techniques, tumor stage and sample size $(4,10)$. GLUT-1 expression has been found to be significantly associated with a reduced response to chemoradiotherapy, in oesophageal cancer, rectal cancer and ovarian carcinoma (9). These differences may be due to variation in histopathological type, immunohistochemical techniques, tumor stage and sample size.

In the present study, the expression and correlations between GLUT-1, P-gp, MRP1 and GST- $\pi$ in laryngeal carcinoma samples was investigated. Whether these proteins are involved in resistance to chemotherapy in patients with laryngeal carcinoma requires further study.

In conclusion, this is the first study to investigate the expression of GLUT-1, P-gp, MRP1 and GST- $\pi$ in laryngeal carcinomas and the correlations between these proteins. P-gp was found to significantly correlate with clinical stage, while MRP1 overexpression was significantly associated with poor survival. In our future studies, we will further investigate whether these proteins may be resistant to chemotherapy in laryngeal carcinoma in vivo. Inhibition of these proteins by targeted treatment may enhance the sensitivity of chemotherapy in laryngeal carcinoma.

\section{Acknowledgements}

This study was supported by the Department of Science and Technology of Zhejiang Provincial (grant no. 2010C33011) and the National Natural Science Foundation of China (grant nos. 81172562 and 81372903).

\section{References}

1. Pérez-Sayáns M, Supuran CT, Pastorekova S, et al: The role of carbonic anhydrase IX in hypoxia control in OSCC. J Oral Pathol Med 42: 1-8, 2013.

2. Hong B, Lui VW, Hashiguchi M, Hui EP and Chan AT: Targeting tumor hypoxia in nasopharyngeal carcinoma. Head Neck 35: $133-145,2013$

3. Gu J, Yamamoto H, Fukunaga H, et al: Correlation of GLUT-1 overexpression, tumor size, and depth of invasion with 18F-2-fluoro-2-deoxy-D-glucose uptake by positron emission tomography in colorectal cancer. Dig Dis Sci 51: 2198-2205, 2006.
4. Wu XH, Chen SP, Mao JY, Ji XX, Yao HT and Zhou SH: Expression and significance of hypoxia-inducible factor- $1 \alpha$ and glucose transporter-1 in laryngeal carcinoma. Oncol Lett 5: 261-266, 2013.

5. Xu YY, Bao YY, Zhou SH and Fan J: Effect on the expression of MMP-2, MT-MMP in laryngeal carcinoma Hep-2 cell line by antisense glucose transporter-1. Arch Med Res 43: 395-401, 2012.

6. Zhou SH, Fan J, Chen XM, Cheng KJ and Wang SQ: Inhibition of cell proliferation and glucose uptake in human laryngeal carcinoma cells by antisense oligonucleotides against glucose transporter-1. Head Neck 31: 1624-1633, 2009.

7. Shimanishi M, Ogi K, Sogabe Y, et al: Silencing of GLUT-1 inhibits sensitization of oral cancer cells to cisplatin during hypoxia. J Oral Pathol Med 42: 382-388, 2013.

8. Chiba I, Ogawa K, Morioka T, et al: Clinical significance of GLUT-1 expression in patients with esophageal cancer treated with concurrent chemoradiotherapy. Oncol Lett 2: 21-28, 2011.

9. Brophy S, Sheehan KM, McNamara DA, Deasy J, Bouchier-Hayes DJ and Kay EW: GLUT-1 expression and response to chemoradiotherapy in rectal cancer. Int J Cancer 125: 2778-2782, 2009.

10. Cantuaria G, Fagotti A, Ferrandina G, et al: GLUT-1 expression in ovarian carcinoma: association with survival and response to chemotherapy. Cancer 92: 1144-1150, 2001.

11. Yu Z, Peng S, Hong-Ming $\mathrm{P}$ and Kai-Feng W: Expression of multi-drug resistance-related genes MDR3 and MRP as prognostic factors in clinical liver cancer patients. Hepatogastroenterology 59: 1556-1559, 2012.

12. Wang J, Zhang J, Zhang L, et al: Expression of P-gp, MRP, LRP, GST- $\pi$ and TopoII $\alpha$ and intrinsic resistance in human lung cancer cell lines. Oncol Rep 26: 1081-1089, 2011.

13. Calatozzolo C, Gelati M, Ciusani E, et al: Expression of drug resistance proteins Pgp, MRP1, MRP3, MRP5 and GST-pi in human glioma. J Neurooncol 74: 113-121, 2005.

14. Wang BL, Zhai HY, Chen BY, et al: Clinical relationship between MDR1 gene and gallbladder cancer. Hepatobiliary Pancreat Dis Int 3: 296-299, 2004.

15. Min L, Chen Q, He S, Liu S and Ma Y: Hypoxia-induced increases in A549/CDDP cell drug resistance are reversed by RNA interference of HIF-1 $\alpha$ expression. Mol Med Rep 5: 228-232, 2012.

16. Lelong-Rebel I, Brisson C, Fabre M, Bergerat JP and Rebel G: Effect of pO2 on antitumor drug cytotoxicity on MDR and non-MDR variants selected from the LoVo metastatic colon carcinoma cell line. Anticancer Res 28: 55-68, 2008.

17. Sobin LH, Gospodarowicz MK and Wittekind C (eds.): TNM Classification of Malignant Tumors. 7th ed. Oxford: Wiley-Blackwell, pp336, 2010.

18. Camenzind RS, Chip S, Gutmann H, Kapfhammer JP, Nitsch C and Bendfeldt K: Preservation of transendothelial glucose transporter 1 and P-glycoprotein transporters in a cortical slice culture model of the blood-brain barrier. Neuroscience 170: 361-371, 2010.

19. Bourasset F, Cisternino S, Temsamani J and Scherrmann JM: Evidence for an active transport of morphine-6-beta-d-glucuronide but not P-glycoprotein-mediated at the blood-brain barrier. J Neurochem 86: 1564-1567, 2003. 